\title{
ROOTS OF THE AFFINE CREMONA GROUP
}

\author{
ALVARO LIENDO
}

\begin{abstract}
Let $\mathbf{k}^{[n]}=\mathbf{k}\left[x_{1}, \ldots, x_{n}\right]$ be the polynomial algebra in $n$ variables and let $\mathbb{A}^{n}=\operatorname{Spec} \mathbf{k}^{[n]}$. In this note we show that the root vectors of the affine Cremona group $\operatorname{Aut}\left(\mathbb{A}^{n}\right)$ with respect to the diagonal torus are exactly the locally nilpotent derivations $\mathbf{x}^{\alpha} \frac{\partial}{\partial x_{i}}$, where $\mathbf{x}^{\alpha}$ is any monomial not depending on $x_{i}$. This answers a question due to Popov.
\end{abstract}

\section{INTRODUCTION}

Letting $\mathbf{k}$ be an algebraically closed field of characteristic zero, we let $\mathbf{k}^{[n]}=\mathbf{k}\left[x_{1}, \ldots, x_{n}\right]$ be the polynomial algebra in $n$ variables, and $\mathbb{A}^{n}=\operatorname{Spec} \mathbf{k}^{[n]}$ be the affine space. The Cremona group $\operatorname{Aut}\left(\mathbb{A}^{n}\right)$ is the group of automorphisms of $\mathbb{A}^{n}$, or equivalently, the group of $\mathbf{k}$-automorphisms of $\mathbf{k}^{[n]}$. We define $\operatorname{Aut}^{*}\left(\mathbb{A}^{n}\right)$ as the subgroup of volume preserving automorphisms i.e.,

$$
\operatorname{Aut}\left(\mathbb{A}^{n}\right)=\left\{\gamma \in \operatorname{Aut}\left(\mathbb{A}^{n}\right) \mid \operatorname{det}\left(\frac{\partial}{\partial x_{i}} \gamma\left(x_{j}\right)\right)_{i, j}=1\right\} .
$$

The groups $\operatorname{Aut}\left(\mathbb{A}^{n}\right)$ and $\operatorname{Aut}\left(\mathbb{A}^{n}\right)$ are infinite dimensional algebraic groups [Sha66, Kam79].

It follows from [BB66, BB67] that the maximal dimension of an algebraic torus contained in $\operatorname{Aut}^{*}\left(\mathbb{A}^{n}\right)$ is $n-1$. Moreover, every algebraic torus of dimension $n-1$ contained in $\operatorname{Aut}^{*}\left(\mathbb{A}^{n}\right)$ is conjugated to the diagonal torus

$$
\mathbf{T}=\left\{\gamma=\operatorname{diag}\left(\gamma_{1}, \ldots, \gamma_{n}\right) \in \operatorname{Aut}^{*}\left(\mathbb{A}^{n}\right) \mid \gamma_{1} \cdots \gamma_{n}=1\right\} .
$$

A k-derivation $\partial$ on an algebra $A$ is called locally nilpotent (LND for short) if for every $a \in A$ there exists $k \in \mathbb{Z}_{>0}$ such that $\partial^{k}(a)=0$. If $\partial: \mathbf{k}^{[n]} \rightarrow \mathbf{k}^{[n]}$ is an LND on the polynomial algebra, then $\exp (t \partial) \in \operatorname{Aut}^{*}\left(\mathbb{A}^{n}\right)$, for all $t \in \mathbf{k}$ [Fre06]. Hence, $\partial$ belongs to the Lie algebra Lie $\left(\operatorname{Aut}^{*}\left(\mathbb{A}^{n}\right)\right)$.

In analogy with the notion of root from the theory of algebraic groups Spr98, Popov introduced the following definition. A non-zero locally nilpotent derivation $\partial$ on $\mathbf{k}^{[n]}$ is called a root vector of $\operatorname{Aut}^{*}\left(\mathbb{A}^{n}\right)$ with respect to the diagonal torus $\mathbf{T}$ if there exists a character $\chi$ of $\mathbf{T}$ such that

$$
\gamma \circ \partial \circ \gamma^{-1}=\chi(\gamma) \cdot \partial, \text { for all } \gamma \in \mathbf{T} \text {. }
$$

The character $\chi$ is called the root of $\operatorname{Aut}^{*}\left(\mathbb{A}^{n}\right)$ with respect to $\mathbf{T}$ corresponding to $\partial$.

Letting $\boldsymbol{\alpha}=\left(\alpha_{1}, \ldots, \alpha_{n}\right) \in \mathbb{Z}_{\geq 0}^{n}$, we let $\mathbf{x}^{\boldsymbol{\alpha}}$ be the monomial $x_{1}^{\alpha_{1}} \cdots x_{n}^{\alpha_{n}}$. In this note we apply the results in [Lie10] to prove the following theorem. This answers a question due to Popov [FR05].

Theorem 1. If $\partial$ is a root vectors of $\operatorname{Aut}^{*}\left(\mathbb{A}^{n}\right)$ with respect to the diagonal torus $\mathbf{T}$, then

$$
\partial=\lambda \cdot \mathbf{x}^{\alpha} \cdot \frac{\partial}{\partial x_{i}}
$$

Date: October 26, 2018.

2010 Mathematics Subject Classification: 14R10, 14R20, 14L30,13N15.

Key words: affine Cremona group, roots of an algebraic group, torus actions, locally nilpotent derivation. 
for some $\lambda \in \mathbf{k}^{*}$, some $i \in\{1, \ldots, n\}$, and some $\boldsymbol{\alpha} \in \mathbb{Z}_{\geq 0}^{n}$ such that $\alpha_{i}=0$. The corresponding root is the character

$$
\chi: \mathbf{T} \rightarrow \mathbf{k}^{*}, \quad \gamma=\operatorname{diag}\left(\gamma_{1}, \ldots, \gamma_{n}\right) \mapsto \gamma_{i}^{-1} \prod_{j=1}^{n} \gamma_{j}^{\alpha_{j}}
$$

\section{Proof of the Theorem}

It is well known that the set Char( $\mathbf{T})$ of characters of $\mathbf{T}$ forms a lattice isomorphic to $M=\mathbb{Z}^{n-1}$. It is customary to fix an isomorphism $M \simeq \operatorname{Char}(\mathbf{T})$ and to denote the character corresponding to $m$ by $\chi^{m}$. The natural $\mathbf{T}$-action on $\mathbb{A}^{n}$ gives rise to an $M$-grading on $\mathbf{k}^{[n]}$ given by

$$
\mathbf{k}^{[n]}=\bigoplus_{m \in M} B_{m}, \quad \text { where } \quad B_{m}=\left\{f \in \mathbf{k}^{[n]} \mid \gamma(f)=\chi^{m}(\gamma) f, \forall \gamma \in \mathbf{T}\right\} .
$$

An LND $\partial$ on $\mathbf{k}^{[n]}$ is called homogeneous if it send homogeneous elements into homogeneous elements. Let $f \in \mathbf{k}^{[n]} \backslash \operatorname{ker} \partial$ homogeneous. We define the degree of $\partial$ as $\operatorname{deg} \partial=\operatorname{deg}(\partial(f))-$ $\operatorname{deg}(f) \in M$ [Lie10, Section 1.2].

Lemma 2. An LND on $\mathbf{k}^{[n]}$ is a root vectors of $\operatorname{Aut}^{*}\left(\mathbb{A}^{n}\right)$ with respect to the diagonal torus $\mathbf{T}$ if and only if $\partial$ is homogeneous with respect to the $M$-grading on $\mathbf{k}^{[n]}$ given by $\mathbf{T}$. Furthermore, the corresponding root is the character $\chi^{\operatorname{deg} \partial}$.

Proof. Let $\partial$ be a root vector of $\operatorname{Aut}^{*}\left(\mathbb{A}^{n}\right)$ with root $\chi^{e}$, so that $\partial=\chi^{-e}(\gamma) \cdot \gamma \circ \partial \circ \gamma^{-1}, \forall \gamma \in \mathbf{T}$. We consider a homogeneous element $f \in B_{m^{\prime}}$ and we let $\partial(f)=\sum_{m \in M} g_{m}$, where $g_{m}$ is homogeneous, so that

$$
\sum_{m \in M} g_{m}=\partial(f)=\chi^{-e}(\gamma) \cdot \gamma \circ \partial \circ \gamma^{-1}(f)=\chi^{-e-m^{\prime}}(\gamma) \sum_{m \in M} \chi^{m}(\gamma) \cdot g_{m}, \quad \forall \gamma \in \mathbf{T}
$$

This equality holds if and only if $g_{m}=0$ for all but one $m \in M$ i.e., if $\partial$ is homogeneous. In this case, $\partial(f)=g_{m}=\chi^{-e-m^{\prime}+m}(\gamma) \cdot \partial(f)$, and so $e=m-m^{\prime}=\operatorname{deg}(\partial(f))-\operatorname{deg}(f)=\operatorname{deg} \partial$.

In [AH06, a combinatorial description of a normal affine $M$-graded domain $A$ is given in terms of polyhedral divisors, and in [Lie10] a description of the homogeneous LNDs on $A$ is given in terms of these combinatorial data in the case where tr. $\operatorname{deg} A=\operatorname{rank} M+1$. In the following we apply these results to compute the homogeneous LNDs on the $M$-graded algebra $\mathbf{k}^{[n]}$. First, we give a short presentation of the combinatorial description in [AH06] in the case where $\operatorname{tr} \cdot \operatorname{deg} A=\operatorname{rank} M+1$. For a more detailed treatment see [Lie10, Section 1.1].

Let $N$ be the dual lattice of $M$. The combinatorial description in [AH06] deals with the following data: A pointed polyhedral cone $\sigma \in N_{\mathbb{Q}}:=N \otimes \mathbb{Q}$ dual to the weight cone $\sigma^{\vee} \subseteq M_{\mathbb{Q}}:=M \otimes \mathbb{Q}$ of the $M$-grading; a smooth curve $C$; and a divisor $\mathfrak{D}=\sum_{z \in C} \Delta_{z} \cdot z$ on $C$ whose coefficients $\Delta_{z}$ are polyhedra in $N_{\mathbb{Q}}$ having tail cone $\sigma$. Furthermore, if $C$ is projective we ask for the polyhedron $\sum_{z \in C} \Delta_{z}$ to be a proper subset of $\sigma$. For every $m \in \sigma^{\vee} \cap M$ the evaluation of $\mathfrak{D}$ at $m$ is the Q-divisor given by

$$
\mathfrak{D}(m)=\sum_{z \in C} \min _{p \in \Delta_{z}} p(m)
$$

We define the $M$-graded algebra

$$
A[\mathfrak{D}]=\bigoplus_{m \in \sigma^{\vee} \cap M} A_{m} \chi^{m}, \quad \text { where } \quad A_{m}=H^{0}\left(C, \mathcal{O}_{C}(\mathfrak{D}(m))\right),
$$

and $\chi^{m}$ is the corresponding character of the torus $\operatorname{Spec} \mathbf{k}[M]$ seen as a rational function on $\operatorname{Spec} A$ via the embedding Frac $\mathbf{k}[M] \hookrightarrow \operatorname{Frac} A[\mathfrak{D}]=\operatorname{Frac} \mathbf{k}(C)[M]$. 
It follows from AH06 that $A[\mathfrak{D}]$ is an normal affine domain and that every normal affine $M$ graded domain $A$ with $\operatorname{tr} . \operatorname{deg} A=\operatorname{rank} M+1$ is equivariantly isomorphic to $A[\mathfrak{D}]$ for some polyhedral divisor on a smooth curve, see also [Lie10, Theorem 1.4].

We turn back now to our particular case where we deal with the polynomial algebra $\mathbf{k}^{[n]}$ graded by Char(T). First, we need to fix an isomorphism $M \simeq \operatorname{Char}(\mathbf{T})$. Let $\left\{\mu_{1}, \ldots, \mu_{n-1}\right\}$ be the canonical basis of $M$. We define the isomorphism $\mu_{i} \mapsto \chi^{\mu_{i}}, i \in\{1, \ldots, n-1\}$, where

$$
\chi^{\mu_{i}}: \mathbf{T} \rightarrow \mathbf{k}^{*}, \quad \gamma=\operatorname{diag}\left(\gamma_{1}, \ldots, \gamma_{n}\right) \mapsto \gamma_{i} .
$$

Since for every $\gamma \in \mathbf{T}$ we have $\gamma_{n}=\gamma_{1}^{-1} \cdots \gamma_{n-1}^{-1}$, the character mapping $\operatorname{diag}\left(\gamma_{1}, \ldots, \gamma_{n}\right) \mapsto \gamma_{n}$ is given by $\chi^{-1}$, where $\mathbb{1}:=\mu_{1}+\ldots+\mu_{n-1}$. Under this isomorphism, the algebra $\mathbf{k}^{[n]}$ is graded by $M$ via $\operatorname{deg} x_{i}=\mu_{i}$, for all $i \in\{1, \ldots, n-1\}$ and $\operatorname{deg} x_{n}=-\mathbb{1}$.

Let now $\left\{\nu_{1}, \ldots, \nu_{n-1}\right\}$ be a basis of $N$ dual to the basis $\left\{\mu_{1}, \ldots, \mu_{n-1}\right\}$ of $M$. We also let $\Delta$ be the standard $(n-1)$-simplex in $N_{\mathbb{Q}}$ i.e., the convex hull of the set $\left\{\nu_{1}, \ldots, \nu_{n-1}, \overline{0}\right\}$.

Lemma 3. The $M$-graded algebra $\mathbf{k}^{[n]}$ is equivariantly isomorphic to $A[\mathfrak{D}]$, where $\mathfrak{D}$ is the polyhedral divisor $\mathfrak{D}=\Delta \cdot[0]$ on $\mathbb{A}^{1}$.

Proof. By [AH06], the $M$-graded algebra $\mathbf{k}^{[n]}$ is isomorphic to $A[\mathfrak{D}]$ for some polyhedral divisor $\mathfrak{D}$ on a smooth curve $C$. Since the weight cone $\sigma^{\vee}$ of $\mathbf{k}^{[n]}$ is $M_{\mathbb{Q}}$, the coefficients of $\mathfrak{D}$ are just bounded polyhedra in $N_{\mathbb{Q}}$.

Since $\mathbb{A}^{n}$ is a toric variety and the torus $\mathbf{T}$ is a subtorus of the big torus, we can apply the method in [AH06, Section 11]. In particular, $C$ is a toric curve. Thus $C=\mathbb{A}^{1}$ or $C=\mathbb{P}^{1}$. Furthermore, the graded piece $B_{\overline{0}} \supsetneq \mathbf{k}$ and so $C$ is not projective by (1). Hence $C=\mathbb{A}^{1}$.

The only divisor in $\mathbb{A}^{1}$ invariant by the big torus is $[0]$, so $\mathfrak{D}=\Delta \cdot[0]$ for some bounded polyhedron $\Delta$ in $N_{\mathrm{Q}}$. Finally, applying the second equation in [AH06, Section 11], a routine computation shows that $\Delta$ can be chosen as the standard $(n-1)$-simplex.

Remark 4. Letting $\mathbb{A}^{1}=\operatorname{Spec} \mathbf{k}[t]$, it is easily seen that the isomorphism $\mathbf{k}^{[n]} \simeq A[\mathfrak{D}]$ is given by $x_{i}=\chi^{\mu_{i}}$, for all $i \in\{1, \ldots, n-1\}$, and $x_{n}=t \chi^{-1}$.

In [Lie10] the homogeneous LNDs on an normal affine $M$-graded domain are classified into 2 types: fiber type and horizontal type. In the case where the weight cone is $M_{\mathbb{Q}}$, there are no LNDs of fiber type. Thus, $\mathbf{k}^{[n]}$ only admits homogeneous LNDs of horizontal type. The homogeneous LNDs of horizontal type are described in [Lie10, Theorem 3.28]. In the following, we specialize this result to the particular case of $A[\mathfrak{D}] \simeq \mathbf{k}^{[n]}$.

Let $v_{i}=\nu_{i}, i \in\{1, \ldots, n-1\}$ and $v_{n}=\overline{0}$, so that $\left\{v_{1}, \ldots, v_{n}\right\}$ is the set of vertices of $\Delta$. For every $\lambda \in \mathbf{k}^{*}, i \in\{1, \ldots, n\}$, and $e \in M$ we let $\partial_{\lambda, i, e}:$ Frac $A[\mathfrak{D}] \rightarrow$ Frac $A[\mathfrak{D}]$ be the derivation given by

$$
\partial_{\lambda, i, e}\left(t^{r} \cdot \chi^{m}\right)=\lambda\left(r+v_{i}(m)\right) \cdot t^{r-v_{i}(e)-1} \cdot \chi^{m+e}, \quad \forall(m, r) \in M \times \mathbb{Z} .
$$

Lemma 5 ([Lie10, Theorem 3.28]). If $\partial$ is a non-zero homogeneous LND of $A[\mathfrak{D}]$, then $\partial=$ $\left.\partial_{\lambda, i, e}\right|_{A[\mathfrak{D}]}$ for some $\lambda \in \mathbf{k}^{*}$, some $i \in\{1, \ldots, n\}$, and some $e \in M$ satisfying $_{j}(e) \geq v_{i}(e)+1, \forall j \neq i$. Furthermore, $e$ is the degree $\operatorname{deg} \partial$.

Proof of Theorem 1. By Lemma 2 the root vectors of $\mathbf{k}^{[n]}$ correspond to the homogeneous LNDs in the $M$-graded algebra $\mathbf{k}^{[n]}$. But the homogeneous LNDs on $A[\mathfrak{D}] \simeq \mathbf{k}^{[n]}$ are given in Lemma 5 , so we only need to translate the homogeneous LND $\partial=\left.\partial_{\lambda, i, e}\right|_{A[\mathfrak{D}]}$ in Lemma 5 in terms of the explicit isomorphism given in Remark 4 .

Let $e=\left(e_{1}, \ldots, e_{n-1}\right)$ and $i \in\{1, \ldots, n-1\}$, so that $v_{i}=\mu_{i}$. The condition $v_{j}(e) \geq v_{i}(e)+1$ yields $e_{i} \leq-1$ and $e_{j} \geq e_{i}+1, \forall j \neq i$. Furthermore, $\partial\left(x_{k}\right)=\partial\left(\chi^{\mu_{k}}\right)=0$, for all $k \neq i, k \in\{1, \ldots, n-1\}$, 


$$
\begin{aligned}
& \partial\left(x_{n}\right)=\partial\left(t \chi^{-\mathbb{1}}\right)=0 \text {, and } \\
& \qquad \begin{array}{l}
\partial\left(x_{i}\right)=\partial\left(\chi^{\mu_{i}}\right)=\lambda t^{-e_{i}-1} \chi^{e+\mu_{i}}=\lambda t^{-e_{i}-1} \chi^{\left(e_{i}+1\right) \mathbb{1}} \chi^{e+\mu_{i}-\left(e_{i}+1\right) \mathbb{1}}=\lambda \mathbf{x}^{\alpha},
\end{array}
\end{aligned}
$$

where $\alpha_{i}=0, \alpha_{n}=-e_{i}-1 \geq 0$, and $\alpha_{k}=e_{k}-e_{i}-1 \geq 0$, for all $k \neq i, k \in\{1, \ldots, n-1\}$. Hence, $\partial=\lambda \cdot \mathbf{x}^{\alpha} \cdot \frac{\partial}{\partial x_{i}}$, for some $\lambda \in \mathbf{k}^{*}$, some $i \in\{1, \ldots, n\}$, and some $\boldsymbol{\alpha} \in \mathbb{Z}_{\geq 0}^{n}$ such that $\alpha_{i}=0$.

Let now $e=\left(e_{1}, \ldots, e_{n-1}\right)$ and $i=n$, so that $v_{n}=0$. The condition $v_{j}(e) \geq v_{n}(e)+1$ yields $e_{j} \geq 1, \forall j \in\{1, \ldots, n-1\}$. Furthermore, $\partial\left(x_{k}\right)=\partial\left(\chi^{\mu_{k}}\right)=0, k \in\{1, \ldots, n-1\}$, and

$$
\partial\left(x_{n}\right)=\partial\left(t \chi^{-1}\right)=\lambda \chi^{e-1}=\lambda \mathbf{x}^{\boldsymbol{\alpha}},
$$

where $\alpha_{n}=0$, and $\alpha_{k}=e_{k}-1 \geq 0$, for all $k \in\{1, \ldots, n-1\}$. Hence, $\partial=\lambda \cdot \mathbf{x}^{\alpha} \cdot \frac{\partial}{\partial x_{n}}$, for some $\lambda \in \mathbf{k}^{*}$, some $i \in\{1, \ldots, n\}$, and some $\boldsymbol{\alpha} \in \mathbb{Z}_{\geq 0}^{n}$ such that $\alpha_{n}=0$.

The last assertion of the theorem follows easily from the fact that the root corresponding to the homogeneous LND $\partial$ is the character $\chi^{\operatorname{deg} \partial}$.

Finally, we describe the characters that appear as a root of $\operatorname{Aut}^{*}\left(\mathbb{A}^{n}\right)$.

Corollary 6. The character $\chi \in \operatorname{Char}(\mathbf{T})$ given by $\operatorname{diag}\left(\gamma_{1}, \ldots, \gamma_{n}\right) \mapsto \gamma_{1}^{\beta_{1}} \ldots \gamma_{n}^{\beta_{n}}$ is a root of Aut ${ }^{*}\left(\mathbb{A}^{n}\right)$ with respect to the diagonal torus $\mathbf{T}$ if and only if the minimum of the set $\left\{\beta_{1}, \ldots, \beta_{n}\right\}$ is achieved by one and only one of the $\beta_{i}$.

Proof. By Theorem 1, the roots of $\operatorname{Aut}^{*}\left(\mathbb{A}^{n}\right)$ are the characters $\operatorname{diag}\left(\gamma_{1}, \ldots, \gamma_{n}\right) \mapsto \gamma_{1}^{\beta_{1}} \cdots \gamma_{n}^{\beta_{n}}$, where $\beta_{i}=-1$ for some $i \in\{1, \ldots, n\}$ and $\beta_{j} \geq 0 \forall j \neq i$. The corollary follows from the fact that $\gamma_{1} \cdots \gamma_{n}=1$.

\section{ACKNOWLEDGMENT}

The author is grateful to Ivan Arzhantsev who pointed out this problem to us.

\section{REFERENCES}

[AH06] Klaus Altmann and Jürgen Hausen. Polyhedral divisors and algebraic torus actions. Math. Ann., 334(3):557607, 2006.

[BB66] A. Białynicki-Birula. Remarks on the action of an algebraic torus on $k^{n}$. Bull. Acad. Polon. Sci. Sér. Sci. Math. Astronom. Phys., 14:177-181, 1966.

[BB67] A. Białynicki-Birula. Remarks on the action of an algebraic torus on $k^{n}$. II. Bull. Acad. Polon. Sci. Sér. Sci. Math. Astronom. Phys., 15:123-125, 1967.

[FR05] G. Freudenburg and P. Russell. Open problems in affine algebraic geometry. In Affine algebraic geometry, volume 369 of Contemp. Math., pages 1-30. Amer. Math. Soc., Providence, RI, 2005.

[Fre06] Gene Freudenburg. Algebraic theory of locally nilpotent derivations. Encyclopaedia of Mathematical Sciences 136. Invariant Theory and Algebraic Transformation Groups 7. Berlin: Springer, 2006.

[Kam79] T. Kambayashi. Automorphism group of a polynomial ring and algebraic group action on an affine space. $J$. Algebra, 60(2):439-451, 1979.

[Lie10] Alvaro Liendo. Affine $\mathbb{T}$-varieties of complexity one and locally nilpotent derivations. Transform. Groups, 15(2):389-425, 2010. Preprint at arXiv:0812.0802v1 [math.AG], 31p.

[Sha66] I. R. Shafarevich. On some infinite-dimensional groups. Rend. Mat. e Appl. (5), 25(1-2):208-212, 1966.

[Spr98] T. A. Springer. Linear algebraic groups, volume 9 of Progress in Mathematics. Birkhäuser Boston Inc., Boston, MA, second edition, 1998.

Mathematisches Institut, Universität Basel, Rheinsprung 21, CH-4051 Basel, Switzerland.

E-mail address: alvaro.liendo@unibas.ch 